УДК 519.218.28

\title{
МАТЕМАТИЧЕСКАЯ МОДЕЛЬ ЭПИДЕМИИ СО СКРЫТЫМ ИНКУБАЦИОННЫМ ПЕРИОДОМ ЗАРАЖЕНИЯ
}

Боровский Андрей Викторович д.ф.-м.Н., профессор ФГБОУ ВО «Байкальский Государственный Университет» кафедра математических методов и цифровых технологий

Галкин Андрей Леонидович д.ф.-м.н.

Институт общей физики им. А.М. Прохорова РАН

\begin{abstract}
Аннотация: рассмотрена математическая модель эпидемии, обладающей скрытым инкубационным периодом заражения. Показано, что в отличие от известных моделей SIR, SIS, SEIR, MSEIR такая эпидемия описывается системой уравнений эпидемической кинетики, содержащей дополнительные слагаемые, учитывающие запаздывание во времени. Решение уравнений эпидемической кинетики с запаздыванием затруднено и возможно только численными методами. Разработан авторский численный метод решения системы уравнений с запаздыванием, который показал хорошую эффективность. Установлен выход решения на квазистационарный режим развития эпидемии.
\end{abstract}

Ключевые слова: теоретические модели эпидемий, вспышка коронавирусной инфекции.

\section{MATHEMATICAL MODEL OF AN EPIDEMIC WITH A LATENT INCUBATION PERIOD OF INFECTION}

A. V. Borovsky

A. L. Galkin

\begin{abstract}
: a mathematical model of an epidemic with a latent incubation period of infection is considered. It is shown that, in contrast to the well-known SIR, SIR, SIR, SIR models, such an epidemic is described by a system of epidemic kinetics equations containing additional terms that take into account the time delay. The solution of the epidemic kinetics equations with a delay is difficult and possible only
\end{abstract}


by numerical methods. The authors developed a numerical method for solving a system of equations with a delay, which showed good efficiency. The output of the solution to the quasi-stationary mode of epidemic development is established.

Key words: theoretical models of epidemics, outbreak of coronavirus infection.

В настоящее время большую актуальность представляет исследование эпидемических моделей. Ссылки на ранние работы имеются в [1]. Вопросы современной эпидемии коронавируса представлены в интернет-статьях [2-3]. Авторы последних, считают, что эпидемия коронавируса плохо поддаётся теоретическому описанию при помощи известных моделей (SIR, SIS, SEIR, MSEIR).

Изучим распространение эпидемии в замкнутой популяции. Примером может служить населённый пункт (город, посёлок, вахтовое производство). Особенностью эпидемии является длительный инкубационный период, предшествующий заболеванию.

Пусть $N_{0}, N_{1}, N_{2}, N_{3}, N_{4}$ - численность популяции, состоящей из здоровых, заражённых, выздоровевших и умерших членов популяции. В соответствии с предположением о замкнутости популяции

$$
\sum_{i=1}^{4} N_{i}(t)=N_{0} \equiv \text { Const }
$$

Далее будем предполагать, что заболевание обладает инкубационным периодом с длительностью $\tau$. К заражению окружающих приводят контакты заболевших, находящихся в инкубационном периоде, со здоровыми людьми, не обладающими иммунитетом. Длительность инкубационного периода для коронавирусной инфекции $\tau=7-10$ дней.

Запишем математическое выражение для скорости уменьшения здоровых членов популяции

$$
\frac{d N_{1}}{d t}(t)=-\kappa^{-}\left|\int_{t-\tau}^{t} \frac{d N_{2}}{d t^{\prime}}\left(t^{\prime}\right) d t^{\prime}\right|-\mathrm{A} .
$$

К заражению здоровых людей приводят члены популяции, которые уже заразились, но находятся в инкубационном периода роста заболевания. Рассматриваемый модельный подход предполагает, что по истечении инкубационного периода человек заболевает в явном виде, медики его 
изолируют и он перестаёт участвовать в заражении окружающих. Поэтому в (2) интегрирование ведётся только по интервалу времени, равному инкубационному периоду. Количество инфицированных членов популяции, участвующих в заражении окружающих, всегда больше нуля и не должно зависеть от направления интегрирования. Поэтому в (2) появляется модуль.

Запишем математическое выражение для скорости изменения количества заражённых членов популяции

$$
\frac{d N_{2}}{d t}(t)=\kappa^{-}\left|\int_{t-\tau}^{t} \frac{d N_{2}}{d t^{\prime}}\left(t^{\prime}\right) d t^{\prime}\right|-\kappa^{+} \int_{0}^{t} \frac{d N_{2}}{d t^{\prime}}\left(t^{\prime}\right) d t^{\prime}-\kappa^{\mathrm{c}} \int_{0}^{t-\tau} \frac{d N_{2}}{d t^{\prime}}\left(t^{\prime}\right) d t^{\prime}+\mathrm{A}
$$

Здесь первое слагаемое описывает скорость появления новых заражённых членов популяции, второе и третье слагаемые описывают убыль количества заражённых вследствие выздоровления и смертности. Коэффициенты $\kappa^{-}, \kappa^{+}, \kappa^{\mathrm{c}}-$ частоты заражения, выздоровления и смертности в расчёте на одного человека. Измеряются в обратных днях.

Скорости увеличения количества выздоровевших и умерших членов популяции определяются выражениями

$$
\frac{d N_{3}}{d t}(t)=\kappa^{+} \int_{0}^{t} \frac{d N_{2}}{d t^{\prime}}\left(t^{\prime}\right) d t^{\prime}, \quad \frac{d N_{4}}{d t}(t)=\kappa^{\mathrm{c}} \int_{0}^{t-\tau} \frac{d N_{2}}{d t^{\prime}}\left(t^{\prime}\right) d t^{\prime} .
$$

Сумма выражений (2)-(4) равна нулю, что является отражением закона сохранения количества членов популяции (1).

Раскроем интегралы в (2-4)

$$
\begin{aligned}
\frac{d N_{1}}{d t}(t)= & -\kappa^{-}\left|N_{2}(t)-N_{2}(t-\tau)\right|-\mathrm{A}, \\
\frac{d N_{2}}{d t}(t)= & \kappa^{-}\left|N_{2}(t)-N_{2}(t-\tau)\right|-\kappa^{+} N_{2}(t)-\kappa^{\mathrm{c}} N_{2}(t-\tau)+\mathrm{A} . \\
& \frac{d N_{3}}{d t}(t)=\kappa^{+} N_{2}(t), \quad \frac{d N_{4}}{d t}(t)=\kappa^{\mathrm{c}} N_{2}(t-\tau) .
\end{aligned}
$$

Постановку задачи (5) следует дополнить условием неотрицательности искомых функций и начальными условиями, например,

$$
N_{i}(t) \geq 0, i=1, . .4, N_{1}(0)=N_{0}, N_{2}(0)=1, N_{3}(0)=0, N_{4}(0)=0 .
$$

Если опустить в (5) слагаемые с запаздыванием $N_{2}(t-\tau)$, то получится модель SIR, известная в эпидемиологии. В этой модели используются другие обозначения - $N_{1}, N_{2}, N_{3} \rightarrow S, I, R$.

В работе [1] система (5) решается аналитически с учётом разложения члена с запаздыванием в ряд Тейлора. 
В данной работе система эпидемической кинетики (5) исследуется численно. Реализована явная разностная схема с запоминанием предыстории к данному моменту уже полученных решений. При шаге 0.1 суток на протяжении всего расчета сумма (1) сохраняется с относительной погрешностью $2,5 \cdot 10^{-6}$.

Коэффициенты в системе (1) приняты равными: частота выздоровления $\kappa^{+}=1 / 15$; частота смертности $\kappa^{c}=1 / 50$; длительность инкубационного периода $\tau=7$ суток; частота заражения (зависит от времени в том числе вследствие возможного принятия карантинных мер)

$$
\kappa^{-}(t)=p(t) \dot{\Delta n}(t)=p(t) \dot{\Delta n_{0}}(t) \frac{N_{1}(t)}{N_{0}}, \quad \sum_{i=1}^{4} N_{i}(t)=N_{0} .
$$

В работе используются следующие выражения для частоты контактов и вероятности заражения, соответствующие плавному введению санитарных норм

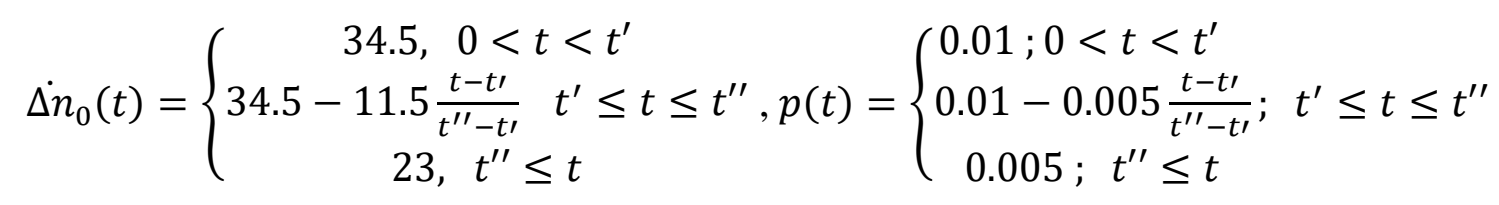

На Рис.1 показан ход эпидемии во времени. ।

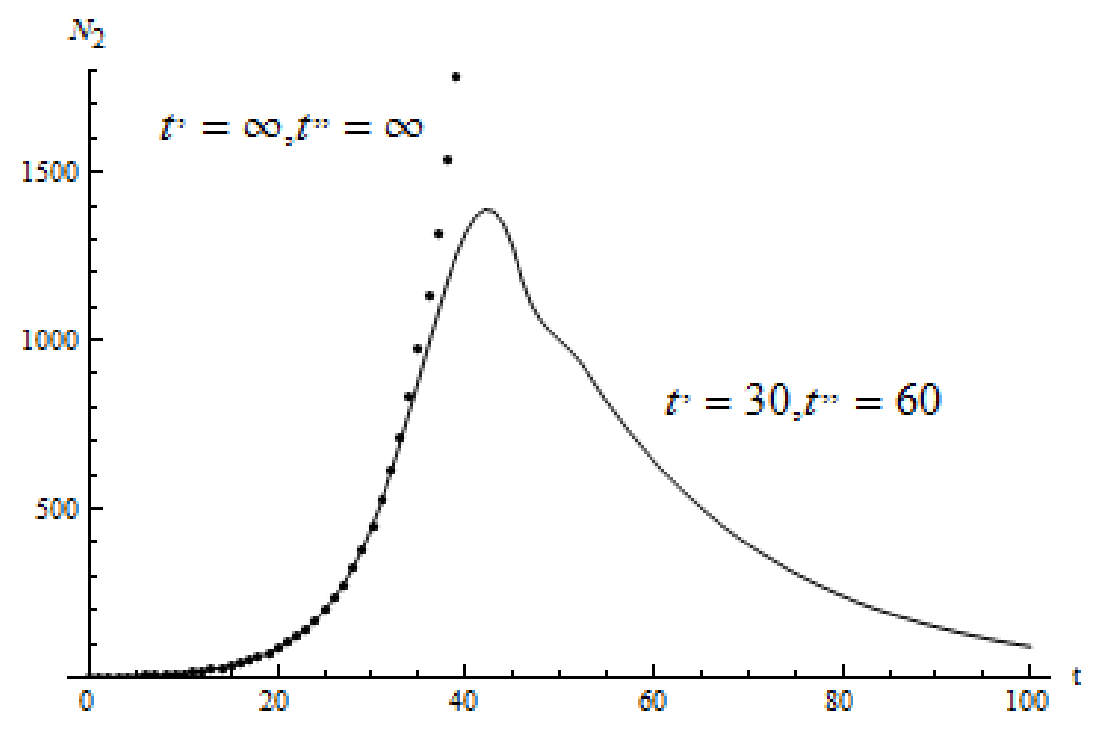

Pис.1. (а) Решение системы (5) без источника ( $\mathrm{A}=0$ ) $N_{0}=6 \cdot 10^{5}$ (Иркутск) с $\mathbf{t}^{\prime}=30, \mathrm{t}^{\prime \prime}=60$ в коэффициентах (6), (7) (сплошная линия). (б)

Развитие эпидемии без введения санитарных мер (точки). 


$$
\frac{d N_{2}}{d t}=0, \quad N_{2}(t)=N_{2}(t-\tau), \quad N_{2}=\frac{A}{\left(k^{+}+k^{c}\right)} .
$$

Источник $\mathrm{A}=$ const $>0$ приводит к постоянному суточному приросту зараженных (9), что может быть использовано для определения величины источника заражения по наиболее достоверной части эпидемической статистики.

Заключение. Получена система уравнений эпидемической кинетики с запаздыванием (5). Исключительно важным для понимания эпидемических процессов является выход решения на квазистационарные значения (8). Наличие постоянного источника заражения после первой волны эпидемии это именно то, что произошло в России и странах Европы. Был открыт выезд туристов на отдых и перестали выполняться санитарные нормы в населённых пунктах. К концу лета люди перестали носить маски, заработали школы, ВУЗы и увеселительные заведения. В результате всплеск эпидемии повторился.

\section{Список литературы}

1. Боровский А.В. Модель эпидемии с запаздыванием. Системный анализ и математическое моделирование. Изд. БГУ, 2020, Т.2, №4.

2. Тамм М. Коронавирусная инфекция в Москве: прогнозы и сценарии https://meduza.io/feature/2020/03/30

3. Иванов М.В. Математическое моделирование процесса пандемии. Теория и практика. https://indsi.ru/2020/04/30 